\title{
The Effectiveness and Reliability of Disclosing Solution in Adhesive Remnant Index: A Pilot Study
}

\author{
Usoff $\mathrm{MF}^{1^{*}}$, Dasor $\mathrm{M}^{2}$, Al-Bayaty $\mathrm{FH}^{3}$ \\ 1. Postgraduate Student DclinDent (Orthodontic), Faculty of Dentistry, University Teknologi MARA, Sg Buloh \\ Campus, 47000 Sg Buloh, Selangor, Malaysia \\ 2. Clinical Specialist (Orthodontics), Centre of Paediatric Dentistry and Orthodontic Studies, Faculty of Dentistry, \\ University Teknologi MARA, Sg Buloh Campus, 47000 Sg Buloh, Selangor, Malaysia \\ 3. Professor, Centre of Periodontolgy Studies, Faculty of Dentistry, University Teknologi MARA, Sg Buloh \\ Campus,47000 Sg Buloh, Selangor, Malaysia
}

*E-mail: drfariq@gmail.com

\begin{abstract}
Introduction: In clinical investigation, scoring the adhesive remnant index (ARI) from tooth surface was difficult due to a lack of a contrasting color in the orthodontic adhesive. Adhesive remnant on the tooth surface is more visible if disclosing solution is applied after debonding. To date, no studies have assessed ARI using a disclosing solution.

Objectives: To compare the effectiveness of visual inspection and digital photography in ARI assessment using disclosing solution and to investigate the reliability of two examiners in scoring ARI on the tooth surface.

Material and Method: Brackets of MBT prescription were bonded to the 10 extracted teeth using self-etching primer and light cure adhesive. All brackets from the samples were mechanically debonded using selected pliers. After debonding, disclosing solution was applied on the adhesive remnant and ARI score were visually recorded. The ARI were also scored from photographs of the samples which were taken with the macro lens of DSLR Camera. Comparison of ARI score between visual and photographic inspection were analyzed using Fisher Exact test. Intra-operator and Inter-operator reliability of ARI score were calibrated using Kappa test.

Result: The Fisher Exact test revealed no statistically significance difference of ARI assessment between visual and photographic inspection ( $p>0.95)$. ARI score of 3 were found $70 \%$ in both techniques. These findings indicated that the bond failure frequently occur between bracket and adhesive. Calibration was carried out and the result showed photographic inspection had a better agreement $(K>0.62)$ than visual inspection $(K>0.57)$ for both calibrations
\end{abstract}

Conclusion: Disclosing solution application is a viable new approach for further clinical study of adhesive remnant on the tooth surface either by visual inspection or photography.

Keywords: adhesive remnant index, debonding, disclosing solution,

\section{Introduction}

In clinical orthodontics, the success of fixed appliance treatment depends on the capability of the adhesive system to resist failure with a large amount of force directed to the bracket or adhesive or enamel as well as to follow easy removal after treatment completed without causing enamel damage. After debonding, the primary Orthodontist's concern is to ensure tooth surface to as near its original state as possible. ${ }^{1}$ The amount of residual adhesive or resin on the surface of the tooth or the bracket base is an important factor for clinicians. Although differences in adhesive remnant scores reflect the bonding strength, adhesive systems that show less residual adhesive are preferable because they are easier and safer to clean up after debonding procedures. However, when less adhesive on tooth surface, it is indicated that the bond failure is on the adhesive-enamel interface. Thus, the tooth is prone to have enamel damage or fracture. The failure should be encouraged within the bracket base/bonding agent interface or within the bonding agent in order to preserve the enamel. ${ }^{2}$ As resin tags penetrate the enamel surfaces, reaching depths up to $50 \mathrm{um},{ }^{3}$ they may exert irreversible effects on the enamel surfaces after orthodontic appliances are removed. Therefore, an assessment system to evaluate the adhesive remnant could be helpful for investigators.

In clinical investigation, orthodontic adhesive had lack of a contrasting colour and it is not easy to detect from naked 
eye. Adhesive remnant on the tooth surface is more visible if a colour agent or dye applies to adhesive remnant after debonding. This approach will facilitate the researcher to score the index accurately. What is the safest agent or dye that can be applied in oral cavity? In preventive dentistry, there is a dye that commonly used known as the disclosing tablets or solution. Its purpose is to detect plaque accumulation on the tooth surface under blue or normal light in dental offices. This selective dye is used to visualize and identify soft debris, pellicle, and bacterial plaque on the surfaces of the teeth. However, it was questionable that the dental disclosing solution is able to detect the adhesive remnant effectively.

Since this solution is no harm to the patient and to date, there is no study to assess Adhesive Remnant Index (ARI) using a disclosing solution, this pilot study is aimed to compare the effectiveness of visual inspection and digital photography in ARI assessment using disclosing solution and to investigate the reliability of two examiners in scoring ARI on the tooth surface.

\section{Material and Methods}

10 extracted teeth mounted on the stone block. Before mounted, the grooves were created on the root surface to make sure the tooth were stable in the stone block during debonding later. The MBT prescription bracket with 0.022 $\mathrm{x} 0.028$ slot were bonded up to the teeth using TransbondTM Plus self-etching primer and TransbondTM XT (3M Unitek) light cure adhesive. All brackets from the samples were mechanically debonded using selected pliers by one operators. Dental disclosing solution (D\&C \#28 1.5\% w/w) was applied on the adhesive remnant and ARI score were recorded using visual inspection. These samples were taken with macro lens (Canon $100 \mathrm{~mm}$ 1:2.8L) of DSLR camera (Canon 70) and the ARI were scored from photographs (Figure 1). Adhesive remnant index (Artun and Bergland 1985) score are:

$0=$ no adhesive left on tooth

$1=$ less than half adhesive left on tooth

$2=$ more than half of the adhesive left on tooth

$3=$ all adhesive left on the tooth with distinct impression of the bracket base

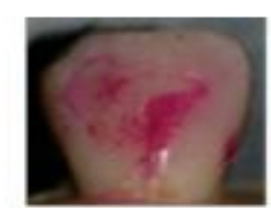

Score 0

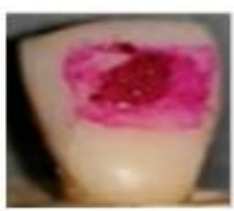

Score 2

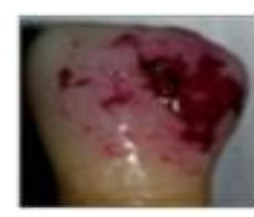

Score 1

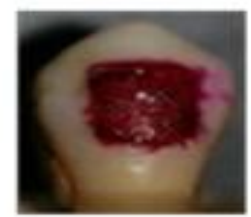

Score 3
Figure 1. Photograph assessment of ARI after application disclosing solution

Comparison of ARI score assessment using photograph and visual inspection were analyzed using Fisher exact test. Intra-operator reliability was assessed by remeasuring ten of the subjects at least 2 times with 2 hours' interval. Inter-operator reliability of the measurement was assessed by specialist for ten debonding brackets. Intraoperator and Inter-operator reliability of ARI score were calibrated using Kappa test and the strength of agreement were decided. ${ }^{4}$ The analysis was carried out using Stata Statistical Software, Version 12.0 (Stata Corporation, College Station, Texas, USA). The level of significance was established at $\mathrm{P}<0.05$.

\section{Results}

The adhesive remnant of ten blocks of teeth has been assessed using disclosing solution after debonding procedure. Table 1 showed the distribution of ARI score of 3 were found $70 \%$ and none on score 0 . These finding indicated that the bond failure frequently occur between bracket and adhesive. Score of 2 and 1 are equally distributed between both assessments. The Fisher exact analysis was performed to compare ARI score between visual inspection and photograph. The result indicated that there were no statistically significance differences of ARI assessment obtained using direct visual and photographs $p$ $(>0.95)$.

Table 1. Fisher exact test to compare visual and photograph assessment between operators

\begin{tabular}{lccccc}
\hline \multirow{2}{*}{$\begin{array}{c}\text { Method of } \\
\text { assessment }\end{array}$} & \multicolumn{5}{c}{ Adhesive Remnant Index Scores } \\
\cline { 2 - 6 } & $\boldsymbol{0}$ & $\boldsymbol{1}$ & $\boldsymbol{2}$ & $\mathbf{3}$ & $\boldsymbol{P ~ V a l u e ~}^{\boldsymbol{a}}$ \\
\hline Visual & $0(0 \%)$ & $2(20 \%)$ & $1(10 \%)$ & $7(70 \%)$ & $\mathrm{P}>0.95$ \\
Photograph & $0(0 \%)$ & $1(10 \%)$ & $2(20 \%)$ & $7(70 \%)$ & \\
\end{tabular}

a: Fisher exact was applied due to assumption of expected value $<5$.

Table 2 showed the result of intra-operator and interoperator reliability of visual and photograph assessment. The strength of agreement is based on the $\mathrm{K}$ value and it can be classified into five categories; 1) poor (less than 
$0.20), 2)$ fair $(0.21-040), 3)$ moderate $(0.41-0.60), 4)$ good (0.61-0.80) and 5) very good (0.81-1.00)4. It is showed that there is moderate agreement in visual inspection between intra-operator and inter-operator which the values are 0.58 and 0.57 , respectively. However, in photographic assessment, the inter-reliability and intra-reliability of the operators showed high level of agreement with 0.62 and 1.00 , respectively.

Table 2. Intra-operator and Inter-operator reliability using Kappa test

\begin{tabular}{ccc}
\hline $\begin{array}{c}\text { Method of } \\
\text { assessment }\end{array}$ & $\begin{array}{c}\text { Value of } \\
\mathbf{K}\end{array}$ & $\begin{array}{c}\text { Strength } \\
\text { of } \\
\text { agreement }\end{array}$ \\
\hline $\begin{array}{c}\text { Visual } \\
\text { (Inter-operator) } \\
\text { Photograph } \\
\text { (Inter-operator) } \\
\text { Visual } \\
\text { (Intra-operator) } \\
\text { Photograph } \\
\text { (Intra-operator) }\end{array}$ & 0.58 & Moderate \\
\hline
\end{tabular}

\section{Discussion}

In this clinical pilot study, the ARI score by Artun and Bergland have been changed to the adhesive remain on the tooth surface instead of from the bracket base. ${ }^{5}$ The adhesive of fixed orthodontic appliances usually is a white or tooth-coloured material. Thus, scoring the adhesive remnant index (ARI) from tooth surface was difficult due to a lack of a contrasting color in the orthodontic adhesive. Adhesive remnant on the tooth surface is more visible when dental disclosing solution is applied after debonding. In this study, we were used the red dye disclosing solution which commonly used in dental practice to detect the plaque accumulation on teeth under the normal light view.

There are several in-vitro studies exist on the assessment of adhesive remnant after bracket debonding. Most of the studies evaluated the bracket base instead the tooth surface. Qualitative or quantitative method have been performed to assess ARI score either on enamel surface or bracket bases such as scanning electron microscopy (SEM), visual inspection, photography, 3-dimensional profilometry, stereomicroscopy and visual inspection with photography under magnification. ${ }^{6-8}$ According to Cehreli et al, there were no significant differences between the qualitative (visual inspection) and quantitative (image analysis on digital photographs) assessment methods when scoring ARI. The visual scoring is capable of generating similar result with the quantitative assessment methods which are more expensive and time consuming. The qualitative method is remained the most frequently used method due it simplicity. ${ }^{6}$

In this clinical pilot study the method for assessing the ARI is by using qualitative assessment methods with dental disclosing solution. The result of this pilot study showed that there is no statistically significance difference between the visual and photograph assessment of ARI. ARI score of 3 were found $70 \%$ in both techniques. These findings indicated that the bond failure frequently occur between bracket and adhesive. This type of bond failure was preferable because less risk of enamel damage during debonding. ${ }^{9}$ It was recommended to have a resin remnant on the tooth surface after debonding to prevent enamel crazing or fracturing. ${ }^{10}$

Furthermore, the results of dental disclosing solution application in ARI were consistent with intra-operator and inter-operator reliability of photograph assessment. There were good agreements (more than 0.62) between two operators in ARI score. These supported $\mathrm{Oz}$ et al. finding that the ARI (4-point scale) score was reliable in evaluation of remnant adhesive. ${ }^{11}$ However, the visual inspection had moderate agreement between two operators. It may be due to the operators who can felt directly the adhesive and gave a different score especially when the adhesive is less or more than half remained. In clinical investigation, the photograph assessment give an advantage in reducing the time of assessment at the chairside but it may lead to underestimate or overestimate the ARI score, especially when the adhesive left more than half (score 2) or less than half (score 1) on the tooth surface. This problem can be solved by using image analysis to get the quantitative result of the adhesive remnant. ${ }^{11}$

\section{Conclusions}

Disclosing solution application is a viable new approach for further clinical study of adhesive remnant on the tooth surface either by visual inspection or photography. The application of disclosing solution may give benefit in clinical investigation due to its simplicity, ease and time saving

\section{Acknowledgment}

My greatest appreciation to my supervisors, colleagues and staff of Faculty of Dentistry, UiTM for their continuous support to ensure the success of this research

\section{References}

1. Campbell PM, Enamel Surface after debonding bracket. Angle Orthodontic; 1995; 65(2) :103-110

2. Retief DH, Failure at the dental adhesive - etched ename interface. Journal of Oral Rehabilitation. 1974; 1: 265-284.

3. Philips RW and Moore BK, Synthetic Resin.In element of dental materials for dental hygienist and dental assistant, Philadephia; 1994

4. Altman DG, Practical statistics for medical research. London: Chapman and Hall; 1991.

5. Artun J, Bergland S. Clinical trial with cystal growth conditioning as an alternative to acid etch enamel pretreatment. American Journal of Orthodontics and Dentofacial Orthopedics. $1984 ; 85(4): 333-40$

6. Cehreli SB, Polat-Ozsoy O, Sar C, Cubukcu HE, Cehrel ZC.A comparative study of qualitative and quantitative methods for the assessment of adhesive remnant after bracket debonding. Eur J Orthod. 2012;34:188-192

7. Kim SS, Park WK, Son WS, Ahn HS, Ro JH, Kim YD.Enamel surface evaluation after removal of orthodontic 
composite remnants by intraoral sandblasting:a 3dimensional surface profilometry. Am J Orthod Dentofacial Orthop.2007;132:71-76.

8. Montasser MA, Drummond JL. Reliability of the adhesiveremnant index score system with different magnifications.Angle Orthod. 2009;79:773-776

9. Northrup RG, Berzins DW, Breadley TG, Schuckit W. Shear bond strength comparison between two orthodontic adhesives and self-ligating and conventional brackets. Angle Orthod 2007;77:701-706

10. Reynolds IR. A review of direct orthodontic bonding. Br J Orthod 1975;2:71-78.

11. O” z AA, Yaziciog lu S, Arici N, Akdeniz BS,Murat N, Arıc1 S. Assessment of the confidence of the adhesive remnant index score with different methods. TurkishJ Orthod. 2013;4:149-153.

12. Kechagia A, Zinelis S, Pandis N, Athanasiou AE, Eliades T. The effect of orthodontic adhesive and bracket-base design in adhesive remnant index on enamel. Journal of the World Federation of Orthodontists 2015;4:18-22

13. Arici, S. Orthodontic bracket (literature review). Turk Ortodonti Dergisi. 1998;11(2): 175-187

14. Bishara, S. E. Comparison of the debonding characteristics of two innovative ceramic bracket designs. American Journal of Orthodontics and Dentofacial Orthopedics. 1999;116 : 86-92.

15. Chen, H. S., Su, M. Z., Chang, H. F. F., Chen Y. J., Lan, W. H., \& Lin, C. P. Effects of different debonding techniques on the debonding forces and failure modes of ceramic brackets in simulated clinical set-ups. American Journal of Orthodontics and Dentofacial Orthopedics. 2007; 132: 680686.

16. Redd, T. B., \& Shivapuja, P.K. Debonding ceramic brackets: effects on enamel. Journal clinical dentistry. 1991 ;25(8): 475-481.

17. Russell, J. S. Current Products and Practice Aesthetic Orthodontic Brackets. Journal of Orthodontics.2005;32: 146-163.

18. Uysal, T., Ustdal, A., \& Kurt, G. Evaluation of shear bond strength of metallic and ceramic brackets bonded to enamel prepared with self-etching primer. European Journal of Orthodontics. 2010; 32: 214-218. 